\title{
Characteristics of Beta-blocker Treatment in Cardiac Patients with Concomitant Chronic Obstructive Pulmonary Disease
}

\author{
Attila Frigy¹, Katalin Mezei², Ildikó Kocsis³ ${ }^{3}$ Lehel Máthé ${ }^{4}$ \\ 1 Department of Internal Medicine IV, University of Medicine and Pharmacy, Tîrgu Mureș, Romania \\ 2 Department of Cardiology, County Emergency Hospital, Miercurea Ciuc, Romania \\ 3 Department of Cardiology, County Emergency Clinical Hospital, Tîrgu Mureș, Romania \\ ${ }^{4}$ Department of Internal Medicine VII, University of Medicine and Pharmacy, Tîrgu Mureș, Romania
}

\section{CORRESPONDENCE}

\section{Attila Frigy}

Str. Gheorghe Marinescu nr. 1

540103 Tîrgu Mureș, Romania

Tel: +40 745653448

E-mail: afrigy68@gmail.com

\section{ARTICLE HISTORY}

Received: 8 October, 2016

Accepted: 6 November, 2016
Katalin Mezei • Str. Doctor Dénes László nr. 2, 530173 Miercurea Ciuc, Romania

Ildikó Kocsis • Str. Gheorghe Marinescu nr. 50, 540136 Tîrgu Mureș, Romania

Lehel Máthé • Str. Gheorghe Marinescu nr. 1, 540103 Tîrgu Mures, Romania

\begin{abstract}
Background: Recent scientific data demonstrated a potential beneficial effect of beta-blocker (BB) therapy in cardiac patients with chronic obstructive pulmonary disease (COPD). Our aim was to characterize the use of beta-blockers in these patients, in "real-life" conditions. Material and methods: We collected retrospectively the data of 60 consecutive cardiac patients (51 men, 9 women, mean age 67 years) with the concomitant diagnosis of COPD: main cardiac conditions, presence and reason of BB therapy, type of drug and dosage, main ECG and echocardiographic parameters, medication and data regarding COPD. Besides descriptive statistics, we compared the data of patients with and without BB therapy (chi-square test, level of significance alpha $<0.05$ ) in order to identify factors associated with BB usage. Results: In our study population, $41.6 \%$ of the patients had received BB treatment, the most frequently used drug being bisoprolol $2.5 \mathrm{mg}$ and $5 \mathrm{mg}$ q.d. (28\%-28\%), followed by carvedilol (32\%). The prevalence of BB therapy was 51.2\% in heart failure patients (48\% in NYHA class III and IV, and $66.6 \%$ in dilated cardiomyopathy), $38.4 \%$ in hypertension, $81.8 \%$ in ischemic artery disease, and $64.7 \%$ in subjects with atrial fibrillation. The usage of BB therapy was significantly associated with the presence of heart failure $(p=0.047)$, dilated cardiomyopathy $(p=0.034)$, ischemic heart disease $(p=0.005)$, previous myocardial infarction $(p=0.003)$ and, inversely, with the acute exacerbation of COPD ( $p=0.006)$. Conclusions: Despite the fact that every cardiac patient with COPD had a potential indication for BB treatment, this was used insufficiently, especially in case of heart failure patients. In daily practice, there is a need for continuous review and improvement of BB usage in these patients.
\end{abstract}

Keywords: beta-adrenergic blockers, heart failure, ischemic heart disease, chronic obstructive pulmonary disease

\section{INTRODUCTION}

Beta-blocker (BB) therapy is commonly used in cardiovascular diseases, the classical indications being hypertension, ischemic heart disease, heart failure, and arrhythmias. Although the perception of BB therapy and its indications 
have been quite crystallized over the years, potential new areas of utilization are still the subject of ongoing research. Recently, one of the observations regarding BB treatment was its - seemingly paradoxical - beneficial effect in case of chronic obstructive pulmonary disease (COPD). ${ }^{1-3}$ This may imply changing the current paradigm, which consists in a relative contraindication of $\mathrm{BB}$ in $\mathrm{COPD}$, due to the fact that they may cause bronchospasm.

Comorbidities are frequent in COPD patients. They include several types of heart disease (close to a prevalence of $60 \%$ ), the most common being hypertension (approximately 50\%), congestive heart failure (20-30\%) and ischemic heart disease (about $25 \%$ ). The coexistence of COPD and heart disease confers a poor prognosis to these patients. For instance, mortality rates for ischemic heart disease are 2-4 times higher in the presence of COPD, and are proportional with the degree of airway obstruction. Furthermore, $50 \%$ of patients with COPD are hospitalized for cardiovascular reasons. ${ }^{4-7}$ Regarding the causes of mortality in patients with COPD, cardiovascular diseases (22\%) occupy the second place after lung cancer $(33 \%){ }^{8}$

Considering the above, we performed a study on the characteristics of $\mathrm{BB}$ usage in patients with concomitant COPD and heart disease, in "real-life" conditions. Also, our aim was to identify factors associated with the presence of BB therapy in these patients.

\section{MATERIAL AND METHODS}

A retrospective data collection was performed using the hospital records and discharge summaries of patients admitted to the Department of Cardiology of the Mures County Emergency Clinical Hospital between 2010 and 2015. Upon admission, all patients signed the general consent form used in our institution, agreeing with anonymous data collection and usage for scientific purposes. Approval of the local ethics committee $(3865 / 01.03 .2016)$ was obtained for confidential data processing and publication.

In 60 consecutive cardiac patients ( 51 men, 9 women, mean age 67 years) with the concomitant diagnosis of COPD, we collected the following data: demographics, main cardiac diagnoses, presence and reason of $\mathrm{BB}$ therapy, type of drug and dosage, main ECG and echocardiographic findings, medication, characteristics of COPD. Besides descriptive statistics, comparison of the data of patients with and without BB therapy was performed using the chi-square test, the level of significance was set at an alpha value of $<0.05$, and the software used for the calculations was GraphPad InStat 3.0.

\section{RESULTS}

In our study population, $41.6 \%$ of the patients received treatment with BBs. Bisoprolol was the most commonly used drug, the dose of $5 \mathrm{mg}$ q.d. and $2.5 \mathrm{mg}$ q.d. occurring in equal proportions $(28 \%-28 \%)$, followed by carvedilol (32\%), the most frequent dosage being $6.25 \mathrm{mg}$ b.i.d., metoprolol (8\%, either tartrate or succinate, most frequently $25 \mathrm{mg}$ b.i.d.) and nebivolol (4\%, $5 \mathrm{mg}$ q.d.).

Heart failure (67\%) was the indication of BB therapy in the majority of cases, followed by hypertension (65\%) and ischemic heart disease (16\%). In our cohort, 35 (85\%) patients with heart failure were classified as NYHA class III and IV. Every patient had at least one cardiac condition with potential indication for BB therapy.

$\mathrm{BB}$ treatment usage was $51.2 \%$ in heart failure, $81.8 \%$ in ischemic heart disease and $38.4 \%$ in case of hypertension. In patients with dilated cardiomyopathy BBs were used in $66.6 \%$ of cases, while in atrial fibrillation (38.3\% from the total number of patients) in $64.7 \%$ of cases.

Patients receiving digoxin (21.6\%) had concomitant BB treatment in $56.2 \%$ of cases, patients on diuretics (68.3\%) in $46.1 \%$ of cases, and those on renin-angiotensin-aldosterone system (RAAS) blocking agents (50\%) in $46.6 \%$ of cases.

More than half of patients (58.3\%) were admitted for an exacerbation of COPD and from those, $22 \%$ had received BB therapy, while $68 \%$ of the stable COPD patients were treated with beta-blockers. The acute cases were administered bisoprolol in $85 \%$ of cases, while the stable patients were treated in equal proportion with both bisoprolol and the other BBs.

The main data of $\mathrm{BB}$ usage and the analysis of association with the presence of $\mathrm{BB}$ treatment are presented in Table 1.

\section{DISCUSSION}

In patients with $\mathrm{COPD}$, the usage of $\mathrm{BBs}$ is generally much lower than it would be desired, given the associated cardiovascular diseases with clear indication for BB treatment - heart failure with reduced ejection fraction, various forms of ischemic heart disease, following a myocardial infarction - e.g. only half of the COPD patients remain on chronic BB therapy after an episode of acute coronary syndrome. ${ }^{9-12}$ In our patients the underutilization of BBs in heart failure was striking. However, in the setting of ischemic heart disease, the BB usage was proper.

The main reason for the under-usage of BBs in daily practice is that it could cause bronchospasm. However, 
TABLE 1. The main data of BB usage in the patient population

\begin{tabular}{lccc}
\hline $\begin{array}{l}\text { COPD associated condition } \\
\text { (\% of patients) }\end{array}$ & $\begin{array}{c}\text { On BB treatment } \\
\text { (\%) }\end{array}$ & $\begin{array}{c}\text { The most used } \\
\text { BB }\end{array}$ & $\begin{array}{c}\text { Association with } \\
\text { BB usage } \\
\text { (p value) }\end{array}$ \\
\hline Heart failure (67\%) & $51.2 \%$ & Bisoprolol & $0.047^{*}$ \\
NYHA III and IV (85\%) & $48 \%$ & & 0.66 \\
Dilated cardiomyopathy (48\%) & $66.6 \%$ & & $0.034^{*}$ \\
Hypertension (65\%) & $38.4 \%$ & Bisoprolol & 0.5 \\
Ischemic heart disease (16\%) & $81.8 \%$ & Bisoprolol & $0.005^{*}$ \\
Previous myocardial infarction (44\%) & & & $0.003^{*}$ \\
Atrial fibrillation (38.3\%) & $64.7 \%$ & Metoprolol & 0.25 \\
Acute exacerbation (58.3\%) & $22 \%$ & Bisoprolol & $0.006^{* *}$ \\
\hline
\end{tabular}

*significant association, ${ }^{* *}$ significant negative association

there are data about the usefulness of maintaining $\mathrm{BB}$ treatment even in the setting of COPD exacerbations. ${ }^{13,14}$ Confirming our expectations, the most important limiting factor of BB treatment in our patients was the presence of COPD exacerbation.

Many studies demonstrated that highly beta 1-selective antagonists (bisoprolol, nebivolol) do not reduce the forced expiratory volume (FEV) in COPD patients, neither in acute nor chronic usage. Also, they do not decrease the effect of beta-agonists; moreover, they counteract their cardiac side effects (tachycardia, arrhythmias, anxiety). In the case of heart diseases with indication for $\mathrm{BB}$ and concomitant COPD, selective BBs proved to have a more consistent beneficial effect than those without beta 1-selectivity. ${ }^{13-17}$

Finally, epidemiological studies and meta-analyses clearly demonstrated in non-selected COPD populations (with or without heart diseases) that BB treatment decreased mortality and acute exacerbations of COPD. ${ }^{1-3,17-20}$ The possible mechanisms of the beneficial effects (cardioprotection mainly) of $\mathrm{BB}$ in the setting of COPD include: the presence of subclinical heart disease (ischemic heart disease, heart failure, arrhythmias etc.), ${ }^{5}$ the presence of clinically manifest heart disease, ${ }^{10}$ the attenuation of cardiovascular side effects of beta-agonist medication, ${ }^{11}$ and the correction of bronchial beta2-receptor down-regulation induced by beta-agonists. ${ }^{21}$

The practical rules of using BBs in cardiac patients with concomitant COPD are as follows: (1) assessing the patient's pulmonary function (spirometry) before starting the treatment; (2) choosing a highly beta 1-selective agent (bisoprolol, nebivolol); and (3) applying the principle of "start low - go slow", as in the case of BB treatment in systolic heart failure, reaching the maximum tolerated dose in 2-week steps of up-titration. ${ }^{21}$ Cessation of therapy should be done if pulmonary function (FEV) clearly deteriorates under treatment. In our patients the highly selective (beta 1 :beta $2=75: 1$, in vitro) bisoprolol was the drug of choice in the majority of patients, the dosage being a moderate one. This finding reflects a comprehensive and pragmatic approach of the treating physicians. ${ }^{22}$

\section{CONCLUSIONS}

In a cohort of patients with concomitant heart disease and COPD we found a general tendency of sub-utilization of BB therapy, mainly in the case of heart failure. Our data underscores the fact that there is a need for reconsideration of $\mathrm{BB}$ therapy in this setting, keeping in mind the proved beneficial effects of BB treatment both in cardiac and COPD patients. Using highly selective $\mathrm{BB}$ agents and a stepwise approach of dosing makes this therapy safe and well tolerated in the case of association of COPD and heart disease.

\section{FUNDING}

No funding was involved in supporting this work.

\section{COMPETING INTERESTS}

None.

\section{REFERENCES}

1. Salpeter S, Ormiston T, Salpeter E. Cardioselective beta-blockers for chronic obstructive pulmonary disease. Cochrane Database Syst Rev. 2005;(4):CD003566

2. Short PM, Lipworth SI, Elder DH, Schembri S, Lipworth BJ. Effect of beta blockers in treatment of chronic obstructive pulmonary disease: a retrospective cohort study. BMJ. 2011;342:d2549.

3. Rutten FH, Zuithoff NP, Hak E, Grobbee DE, Hoes AW. Beta-blockers may reduce mortality and risk of exacerbations in patients with chronic obstructive pulmonary disease. Arch Intern Med. 2010;170:880-887. 
4. Hawkins NM, Petrie MC, Jhund MC, Chalmers GW, Dunn FG, McMurray JJV. Heart failure and chronic obstructive pulmonary disease: diagnostic pitfalls and epidemiology. Eur J Heart Fail. 2009;11:130-139.

5. Maclay D, MacNee W. Cardiovascular disease in COPD: Mechanisms. Chest. 2013;143:798-807.

6. Andell P, Koul S, Martinsson A, et al. Impact of chronic obstructive pulmonary disease on morbidity and mortality after myocardial infarction. Open Heart. 2014;1:e000002.

7. Díez JM, Morgan JC, García RJ. The association between COPD and heart failure risk: a review. Int J Chron Obstruct Pulmon Dis. 2013;8:305-312.

8. Zvezdin B, Milutinov S, Kojicic M, et al. A postmortem analysis of major causes of early death in patients hospitalized with COPD exacerbation. Chest. 2009:136:376-380.

9. Lipworth B, Skinner D, Devereux G, et al. Underuse of $\beta$-blockers in heart failure and chronic obstructive pulmonary disease. Heart. doi:10.1136/ heartjnl-2016-309458.

10. Etminan M, Jafari S, Carleton B, Fitzgerald JM. Beta-blocker use and COPD mortality: a systematic review and meta-analysis. BMC Pulm Med. 2012;12:48.

11. Albouaini K, Andron M, Alahmar A, Egred M. Beta-blockers use in patients with chronic obstructive pulmonary disease and concomitant cardiovascular conditions. Int J Chron Obstruct Pulmon Dis. 2007;2:535540

12. Stone IS, Barnes NC, Petersen SE. Chronic obstructive pulmonary disease: a modifiable risk factor for cardiovascular disease? Heart. 2012;98:10551106.

13. Chang CL, Mills GD, McLachlan JD, Karalus NC, Hancox RJ. Cardioselective and non-selective beta-blockers in chronic obstructive pulmonary disease: effects on bronchodilator response and exercise. Intern Med J. 2010;40:193-200
14. Stefan MS, Rothberg MB, Priya A, Pekow PS, Au DH, Lindenauer PK. Association between $\beta$-blocker therapy and outcomes in patients hospitalized with acute exacerbations of chronic obstructive lung disease with underlying ischemic heart disease, heart failure or hypertension. Thorax. 2012;67:977-984

15. Su VY, Chang YS, Hu YW, Hung MH, et al. Carvedilol, bisoprolol, and metoprolol use in patients with coexistent heart failure and chronic obstructive pulmonary disease. Medicine (Baltimore). 2016;95:e2427.

16. Kubota $Y$, Asai K, Furuse $E$, et al. Impact of $\beta$-blocker selectivity on longterm outcomes in congestive heart failure patients with chronic obstructive pulmonary disease. Int J Chron Obstruct Pulmon Dis. 2015;10:515-523.

17. Quint JK, Herrett E, Bhaskaran K, et al. Effect of $\beta$ blockers on mortality after myocardial infarction in adults with COPD: population based cohort study of UK electronic healthcare records. BMJ. 2013;347:f6650.

18. Andell P, Erlinge D, Smith JG et al. Beta-Blocker use and mortality in COPD patients after myocardial infarction: A Swedish Nationwide Observational Study. J Am Heart Assoc. 2015;4:e001611.

19. Ni Y, Shi G, Wan $H$. Use of cardioselective beta-blockers in patients with chronic obstructive pulmonary disease: a meta-analysis of randomized, placebo-controlled, blinded trials. J Int Med Res. 2012;40:2051-2065.

20. Farland MZ, Peters CJ, Williams JD, Bielak KM, Heidel RE, Ray SM. $\beta$-Blocker use and incidence of chronic obstructive pulmonary disease exacerbations. The Annals of Pharmacotherapy. 2013;47:651-656.

21. Campo G., Pavasini R, Biscaglia S, Contoli M, Ceconi C. Overview of the pharmacological challenges facing physicians in the management of patients with concomitant cardiovascular disease and chronic obstructive pulmonary disease. Eur Heart J - Cardiovasc Pharmacother. 2015;1:205211.

22. Wellstein A, Palm D, Belz GG. Affinity and selectivity of beta-adrenoceptor antagonists in vitro. J Cardiovasc Pharmacol. 1986;8Suppl11:S36-S40. 